\title{
SPRACHWISSENSCHAFT
}

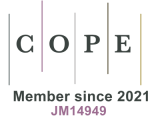

https://doi.org/10.18778/2196-8403.2021.09

JIYE DUAN

JAROSŁAW APTACY

\section{Eine vergleichende Studie zu Verwandtschafts- bezeichnungen im Chinesischen und im Deutschen}

Die Forschung im Bereich der Verwandtschaftsbezeichnungen ist ein wichtiger Bestandteil der Lexikologie und der sozialen Kultur. In diesem Beitrag werden deutsche und chinesische Verwandtschaftsbezeichnungen definiert, es werden ihre Merkmale erschlossen und ihre Semantik dargestellt, um die Bezeichnungen in den beiden Sprachen zu vergleichen. Dadurch wird auch versucht, die Unterschiede zwischen der chinesischen und der deutschen Kultur in diesem Bereich zu identifizieren und deren mögliche Gründe zu ermitteln.

Schlüsselwörter: Verwandtschaftsbezeichnung, Deutsch, Chinesisch, Kulturunterschiede, Wortfeld

\section{A comparative study of kinship terms in Chinese and German}

Research of kinship terms is an important part of lexicology and of social culture studies. This article defines German and Chinese kinship terms, determines their characteristics and presents their semantics in order to compare the terms in the two languages. The Author attempts to identify key differences between Chinese and German culture in this respect and to determine their underlying causes.

Keywords: kinship term, German, Chinese, cultural differences, lexical field 


\section{Nazwy pokrewieństwa w języku chińskim i niemieckim - studium porównawcze}

Badania nad nazwami pokrewieństwa są ważną częścią leksykologii i kultury społeczeństw. W niniejszym artykule zdefiniowano niemieckie i chińskie nazwy pokrewieństwa, określono ich cechy i przedstawiono ich semantykę w celu ich porównania w obu językach. Ponadto podjęto próbę identyfikacji różnic między kulturą chińską i niemiecką w tym obszarze oraz ustalić ich możliwe przyczyny.

Słowa klucze: nazwy pokrewieństwa, język niemiecki, język chiński, różnice kulturowe, pole wyrazowe

\section{Grundlegende Definitionen}

Deutschland und China weisen zahlreiche kulturelle Unterschiede auf, da die beiden Länder zu verschiedenen kulturellen, ja zivilisatorischen, Kreisen gehören. Durch die Analyse der Systeme der Verwandtschaftsnamen in den beiden Sprachen können daher nicht nur die Unterschiede aus sprachwissenschaftlicher Sicht dargestellt, sondern auch die Unterschiede zwischen den beiden Kulturen verstanden werden. Auf Grundlage von MURDOCKS (1949) Klassifikation von Verwandtschaftsbezeichnungen in verschiedenen Sprachgemeinschaften kann gefolgert werden, dass die deutschen und anderen westeuropäischen Verwandtschaftsbezeichnungen dem Eskimo-System angehören. ${ }^{1}$ Das Verwandtschaftsbezeichnungssystem basiert auf der Kern- oder Nuklearfamilie bzw. auf den linearen Verwandten und die kollateralen Verwandten werden nur vage klassifiziert. Zum Beispiel werden die Brüder sowohl des Vaters als auch der Mutter heute gemeinsam als Onkel bezeichnet. Die Schwestern sowohl des Vaters als auch der Mutter werden gemeinsam als Tante bezeichnet. Das war nicht immer so, denn früher nannte man Onkel mütterlicherseits Oheime und Schwestern Muhmen. ${ }^{2}$ Die vorliegende Studie besitzt aber vorrangig synchronen Charakter, so dass auf frühere Sprachzustände nur marginal verwiesen wird, vor allem wenn dies zum Verständnis der heutigen Zustände beitragen soll.

Das chinesische Verwandtschaftsbezeichnungssystem gehört zum sudanesischen Verwandtschaftssystem, das auf männlicher Macht basiert. Ein Merkmal dieses Systems ist eine strikte Unterscheidung zwischen Verwandten väterlicherseits und mütterlicherseits, zum Beispiel heißen die Brüder des Vaters 伯

Vgl. auch Leitfaden für die Einführungsvorlesung in Ethnosoziologie (unibe.ch) (23.08.2021).

2 Vgl. die entsprechenden Einträge: Oheim - Schreibung, Definition, Bedeutung, Etymologie, Synonyme, Beispiele | DWDS, Muhme - Schreibung, Definition, Bedeutung, Etymologie, Synonyme, Beispiele | DWDS (23.08.2021). 
伯 (bóbo ${ }^{3}$, älterer Bruder des Vaters) und 叔叔 (shūshu, jüngerer Bruder des Vaters) und die Schwester des Vaters 姑姑 (gūgū, Schwester des Vaters). Im Gegensatz dazu heißen die Brüder der Mutter 舅舅 (jiùjiu, Bruder der Mutter) und die Schwester der Mutter 姨 (yí, Schwester der Mutter) (vgl. Di 2014:21). Das Verwandtschaftssystem unterscheidet sich in einigen Provinzen Chinas vom sudanesischen Verwandtschaftssystem und gehört zum Verwandtschaftssystem der Irokesen. Im Dialekt der Provinz Shaanxi heißen die Brüder des Vaters beispielsweise auch Vater und vor der Bezeichnung steht normalerweise auch der Rang in der Reihenfolge der Onkel, zum Beispiel heißt der zweitälteste Onkel väterlicherseits 二爸 (èrbà, zweitältester Bruder des Vaters). Diese besondere Situation zeigt, dass sich die Verwandtschaftsbezeichnungssysteme in einem Land ebenfalls voneinander unterscheiden können und jede Region ihre eigenen lokalen historischen Entwicklungsmerkmale aufweisen kann. Daher basieren die in diesem Beitrag diskutierten chinesischen und deutschen Verwandtschaftsbezeichnungssysteme auf den in der modernen chinesischen und deutschen Gesellschaft gebräuchlichen Verwandtschaftsbezeichnungen und -systemen, wobei die Verwendung des Vokabulars für dialektale Bezeichnungen so weit wie möglich vermieden wird.

Der Begriff 亲属 (qīnshǔ, Verwandte(r)) im Chinesischen besteht aus zwei Morphemen, 亲 (qīn, verwandt) und 属 (shǔ, Clan). 亲(qīn, verwandt) bedeutet jemanden, mit dem das Ego eine Blutsverwandtschaft oder eine Ehebeziehung hat. 属 (shǔ, Clan) bezieht sich auf dieselbe Familie und denselben Clan, daher bezieht sich der Begriff 亲属 (qīnshǔ, Verwandte(r)) im Chinesischen auf Personen, die durch Abstammung oder durch Ehe in derselben Familie verwandt oder verschwägert sind, und diese Definition stimmt mit der von 亲者, 属也 (qīn zhě, shǔ yě, die Verwandten gehören zu dem Clan) im Buch der Riten überein. Die Definition im Buch der Riten spiegelt auch das langjährige patriarchalische System Chinas wider. Das patriarchalische System ist ein System, das sich auf die gesamte Familie in der alten chinesischen Gesellschaft erstreckt, Verwandte nach der Entfernung der Blutsverwandtschaft unterscheidet, Clanmitglieder verwaltet und behandelt (vgl. Hu 2007:366).

Im Laufe der Zeit wurde der Umfang des Konzepts 亲属 (qīnshǔ, Verwandte(r)) im Chinesischen jedoch immer größer und erweiterte sich allmählich von der traditionellen Blutsverwandtschaft und Ehebeziehung bis zur Adoptionsbeziehung im rechtlichen Sinne. Diese Art der Änderung tritt auch in Wörterbüchern

3 Die Diakritika weisen auf einen der vier Töne des Chinesischen hin, die in der Pinyin-Transkription üblich sind. 
auf. Das chinesische Wörterbuch definiert 亲属 (qīnshǔ, Verwandte(r)) als Menschen, die durch Blut oder Ehe verwandt/verschwägert sind, insbesondere solche, die durch Blut verwandt sind. ${ }^{4}$ Im Mandarin-Wörterbuch hat der Begriff 亲属 (qīnshǔ, Verwandte(r)) eine andere Definition, die Personen nennt, die durch Blut, Heirat oder Adoption eine Beziehung zueinander haben, wie zum Beispiel Schwiegereltern und Ehepartner. ${ }^{5}$ Das Mandarin-Wörterbuch bezieht auch adoptierte Kinder in den Bereich der Verwandten ein und ist nicht länger auf die traditionelle Blutsverwandtschaft und die Ehebeziehung beschränkt.

Die gesamte Struktur, die durch Blutsverwandtschaft und Schwägerschaft aufgebaut wurde, wird in China 宗族 (zōngzú, Clan) ${ }^{6}$ genannt. Das Clansystem fordert die Chinesen nachdrücklich auf, alle Verwandten nach Geschlecht, Grad der Entfernung sowie nach Alter u.a. zu klassifizieren und unterschiedliche Bezeichnungen zu verwenden, um sie so zu benennen. Daher ist das chinesische Verwandtschaftsbezeichnungssystem sehr komplex und vielschichtig. Aus der Perspektive der Art der Verwandtschaft kann sie in Blutsverwandtschaft und Schwägerschaft unterteilt werden. Je nachdem, ob die Blutsverwandtschaft direkt ist oder nicht, werden die Blutsverwandten nach HU (2007:4) in lineare Verwandte und kollaterale Verwandte unterteilt und Schwiegerverwandte in lineare Schwiegerverwandte und kollaterale Schwiegerverwandte. Aus Sicht des patriarchalischen Systems wird die Verwandtschaft in 宗亲 (zōngqīn, Clanverwandte(r)), 外亲 (wàiqīn, mütterliche Verwandtschaft) und 妻亲 (qīīin, Ehefrauen-Verwandtschaft) unterteilt. Diese komplizierte Methode der Verwandtschaftsklassifizierung hängt auch eng mit 有序(yǒuxù, ordentlich) zusammen, ein Prinzip, an das die Chinesen gewöhnt sind.

Die Definitionen der Verwandtschaft sind im Deutschen recht unterschiedlich. Im Duden Online-Wörterbuch wird Verwandtschaft als eine „Gesamtheit der Beziehungen, Angehörigen, die jemand hat" erklärt. ${ }^{7}$ Die Definition von Verwandtschaft nach Duden ist der Definition im Buch der Riten sehr ähnlich. Beide definieren den Umfang der Verwandtschaft aus einer Makroperspektive und definieren Verwandtschaft als ein Ganzes, das sich aus Verwandten zu-

Vgl. https://www.zdic.net/hans/亲属 (12.05.2021) Die chinesischen Definitionen in der Arbeit wurden von den Autoren ins Deutsche übersetzt.

5 Vgl. https://cidian.18dao.net/zh-hans/zici/親屬 (12.05.2021).

6 Die Clan-Verwandtschaft bezieht sich auf Verwandte, die durch Blut unter demselben Clan bzw. demselben väterlichen Vorfahren verwandt sind (vgl. BAO / WANG 1988:207).

7 Vgl. https://www.duden.de/rechtschreibung/Verwandtschaft (12.05.2021). 
sammensetzt. In der Soziologie werden Verwandte als ein Verantwortlicher Kooperations- und Solidaritätsverband definiert, und es wird darauf hingewiesen, dass diese Art von Verband durch das Erbschaftsrecht, das Inzesttabu und durch Rollenbezeichnungen (Tante, Neffe, Schwager) beschränkt wird (vgl. NAVEHERZ 1998:288, zit. nach JAKOBY 2008:20). HILLMANN (vgl. 1994:909, zit. nach JAKOBY 2008:20) definiert die Verwandtschaft als Bindung zwischen mehreren Personen aufgrund gemeinsamer Abstammung bzw. Vorfahren (Eltern, Großeltern usw.) und infolge von Eheschließungen. Anders als Dudens Definition glaubt er nicht, dass „Verwandtschaft“ ein Ganzes sei, sondern eine Art Verbindung, Relation wie eine unsichtbare Linie, die die Menschen mit einem gemeinsamen Vorfahren oder durch die Ehe verbindet.

Nach Artikeln 1589 und 1590 des Bürgerlichen Gesetzbuches (BGB) werden Verwandtschaft und Schwägerschaft allgemein wie folgt beschrieben:

$\S \S 1589$ BGB (Verwandtschaft)

Personen, deren eine von der anderen abstammt, sind in gerader Linie verwandt. Personen, die nicht in gerader Linie verwandt sind, aber von derselben dritten Person abstammen, sind in der Seitenlinie verwandt. Der Grad der Verwandtschaft bestimmt sich nach der Zahl der sie vermittelnden Geburten.

$\S \S 1590$ BGB (Schwägerschaft)

I Die Verwandten eines Ehegatten sind mit dem anderen Ehegatten verschwägert. Die Linie und der Grad der Schwägerschaft bestimmen sich nach der Linie und dem Grade der sie vermittelnden Verwandtschaft.

II Die Schwägerschaft dauert fort, auch wenn die Ehe, durch die sie begründet wurde, aufgelöst ist.

Zusammenfassend wird Verwandtschaft im deutschen Kontext auch unterteilt in: „Verwandtschaft aufgrund von Abstammung (Deszendenz) und de[n] über Heirat gegründete[n] Verwandtenkreis (Schwägerschaft, Affinalverwandtschaft)“ (JAKOBY 2008:20).

Die Definition von Schwägerschaft nach $\S 1590$ des BGB besagt jedoch, dass die durch die Ehe festgelegten Schwägerschaften auch bei Beendigung der Ehe unverändert bleiben, was mit der chinesischen Tradition nicht übereinstimmt. Wenn eine Ehe endet, wird im Allgemeinen auch die durch die Ehe aufgebaute Schwägerschaft beendet, anstatt unverändert zu bleiben. Da es sich bei der Schwägerschaft um eine rechtliche Verwandtschaft handelt, die die Ehepartner nach ihrer Heirat herbeigeführt haben, ist diese Art der Verwandtschaft im rechtlichen Sinne nicht gesetzlich geschützt und anerkannt, nachdem die Ehe beendet ist, auch wenn die Beziehung zwischen den beiden Parteien noch eng ist. JAKOBY (2008:21) ist auch der Meinung, dass sich Heirat als konstituie- 
rendes Prinzip von verwandtschaftlichen Beziehungen von blutsverwandtschaftlichen Beziehungen vor allem dadurch unterscheidet, dass sie beendet bzw. verändert werden kann. Zudem zitiert JAKOBY (2008:21) die Ansicht von ALLAN (1979) über die Ehe und Schwägerschaft: Heirat als menschliche Konstruktion bzw. soziales Arrangement ist auflösbar und damit einhergehend ebenso die Verwandtschaftsbeziehungen.

Nach dem chinesischen Wörterbuch ist 称谓 (chēngwèi, Bezeichnung) eine Art Name, der die gegenseitigen Beziehungen von Menschen aufgrund von Verwandtschaft oder aufgrund von Identität, Beruf und anderen Aspekten beschreibt und dessen zwei Morpheme 称 (chēng) und 谓 (wèi) die Bedeutung von ,benennen, bezeichnen' aufweisen. 亲属称谓 (qīnshǔ chēngwèi, Verwandtschaftsbezeichnung) bezieht sich auf die Bezeichnung und den Namen, welche die auf Blutsverwandtschaft und Schwägerschaft basierende Beziehung zwischen Clanmitgliedern und dem Ego beschreiben. Zusammenfassend ist es die Art und Weise, Menschen anzusprechen, die miteinander verwandt sind (Hu 2007:1).

\section{Verwendung der deutschen und chinesischen Verwandtschafts- bezeichnungen}

HU (2007:17ff.) weist darauf hin, dass die Verwendung chinesischer Verwandtschaftsbezeichnungen die Einhaltung bestimmter Regeln erfordert, nämlich 面称 (miànchēng, engl. Face-to-Face addressing) und 背称 (bèichēng, engl. back-appellation, back-appellation wird in der Situation verwendet, wenn der Verwandte nicht anwesend ist), 直系宗亲称谓 (zhíxì zōngqīn chēngwèi, lineare Clan-Verwandtschaftsbezeichnung) und 非直系宗亲称谓 (fēi zhíxì zōngqīn chēngwèi, kollaterale Clan-Verwandtschaftsbezeichnung), ${ }^{8}$ 谦称 (qiānchēng, bescheidene Bezeichnung, bescheidene Bezeichnung wird verwendet, um sich selbst oder einen Verwandten höflich anzusprechen) und 尊称 (zūnchēng, respektable Bezeichnung, respektable Bezeichnung wird verwendet, um die andere Partei oder einen Verwandten der anderen Partei mit Respekt

8 Der Unterschied zwischen der linearen Clan-Verwandtschaftsbezeichnung und der kollateralen Clan-Verwandtschaftsbezeichnung besteht normalerweise darin, dass im Fall von back-appellation einigen kollateralen Clan-Verwandtschaftsbezeichnungen ein Unterscheidungselement vorangestellt wird, das die Beziehung angibt, zum Beispiel (岳)父 (yuèfù, Schwiegervater) und (岳)母 (yuèmǔ, Schwiegermutter). 
anzusprechen.), 通语称谓 (tōngyǔ chēngwèi, universale Bezeichnung, die universale Bezeichnung ist die im chinesischen Sprachraum gebräuchliche Bezeichnung) und 方言称谓(fāngyán chēngwèi, dialektale Bezeichnung, die dialektale Bezeichnung stammt aus einem bestimmten Dialekt oder einer Dialektgruppe), 通用称谓 (tōngyòng chēngwèi, allgemeine Bezeichnung, die allgemeine Bezeichnung kann von den meisten Menschen unter normalen Umständen verwendet werden) und 专用称谓 (zhuānyòng chēngwèi, spezielle Bezeichnung, die spezielle Bezeichnung wird von bestimmten Personengruppen bei bestimmten Anlässen verwendet), 古代称谓 (gǔdài chēngwèi, alte Bezeichnung) und 现 代称谓 (xiàndài chēngwèi, moderne Bezeichnung).

Die chinesischen Verwandtschaftsbezeichnungen werden stark von den Traditionen der Altershierarchie in der chinesischen Gesellschaft und der Clankultur beeinflusst. Beispielsweise können die meisten Verwandtschaftsbezeichnungen im Fall von Back-Appellation verwendet werden, aber im Fall von Faceto-Face Addressing sind einige Verwandtschaftsbezeichnungen nur für ältere Verwandte geeignet und für die jüngeren Verwandten werden im Allgemeinen Vornamen oder Spitznamen verwendet, weil es in China als respektlos angesehen wird, ältere Menschen mit Vornamen anzusprechen. Ältere Menschen nennen die jüngeren Leute jedoch normalerweise beim Vornamen oder Spitznamen, um die enge Beziehung zu ihnen zu zeigen. Wenn Chinesen mit Nicht-Verwandten chatten und ihre eigenen Verwandten erwähnen, verwenden sie die Verwandtschaftsbezeichnungen mit neutralen oder abwertenden Präfixen, um die andere Partei zu respektieren. Andererseits verwenden sie bei der Anrede der Verwandten der anderen Partei Bezeichnungen, die positive Affixe enthalten oder positive Bedeutungen aufweisen, zum Beispiel heißen die Eltern des Ego 家父/家母 (jiāfù/jiāmǔ, Vater/Mutter) und die Eltern des anderen 令尊 /令堂 (lìngzūn/lìngtáng, Vater/Mutter der anderen Partei). Dieser Kontrast spiegelt die 谦逊 (qiānxùn, Demut) in der chinesischen Kultur wider, die auch Sapirs Hypothese entspricht, nach der verschiedene Sprachen die einzigartige Art der Erkenntnis von Menschen ausdrücken, die diese Sprache benutzen, d.h., Unterschiede in der Sprache spiegeln Unterschiede im Denken und in der Kultur wider (vgl. GAO 2009:78).

Im Vergleich dazu ist die Verwendung deutscher Verwandtschaftsbezeichnungen im täglichen Leben nicht so umständlich wie die Verwendung chinesischer Verwandtschaftsbezeichnungen. Beispielsweise können jüngere Leute auch Namen von älteren Menschen nennen und es gibt kein lexikalisch und grammatisch ausdrückbares Konzept bescheidener und respektabler Bezeichnungen von Älteren. Es ist erwähnenswert, dass es einen großen Unterschied in der 
Verwendung von Verwandtschaftsbegriffen in beiden Sprachen gibt, d.h., ein Teil von Verwandtschaftsbezeichnungen im Chinesischen kann im täglichen Leben auf Menschen angewendet werden, die nicht verwandt sind. Beispielsweise können ältere Menschen als „Großeltern“, Menschen mittleren Alters als „Onkel und Tanten“ usw. bezeichnet werden. Solche Art von Verwandtschaftsbezeichnung wird als 拟亲属称谓 (nǐ qīnshǔ chēngwèi, Pseudoaffin-Bezeichnung) bezeichnet. Der Grund für diese Situation liegt in 泛伦理化 (fàn lúnlǐhuà, Pan-Ethikalisierung), 家国同构 (jiāguó tónggòu, gleiche Struktur von Familie und Land) im alten China und auch der einzigartigen traditionellen Kultur (vgl. WANG 2007:216). Gleichzeitig hilft eine Pseudo-Verwandtschaftsbezeichnung dabei, Lücken in Namen von sozialen Beziehungen der chinesischsprachigen Gesellschaft zu füllen. In der deutschen sozialen Kommunikation wird jedoch die Verwendung von Verwandtschaftsbezeichnungen für Nichtverwandte vermieden und stattdessen wird Name oder Beruf der Person verwendet. ${ }^{9}$

\section{Vergleichende Analyse}

In diesem Beitrag wird hauptsächlich Verwandtschaft im traditionellen Sinne diskutiert, d.h. die Blutsverwandtschaft. Der Grund für den Ausschluss von Schwägerschaft ist, dass der Vergleich aller relativen Begriffe recht kompliziert und vielfältig ist und er würde den Rahmen dieses Artikels sprengen. Gleichzeitig sind die Blutsverwandtschaftsbezeichnungen repräsentativer, da innerhalb des Alltagslebens die Häufigkeit der Verwendung der Blutsverwandtschaftsbezeichnungen die Grundbedürfnisse decken kann.

In diesem Kapitel wird die lineare Blutsverwandtschaftsbezeichnungskarte von Hu (2007:92) verwendet, die sich auf das Ego und auf die auf- und absteigenden fünf Generationen der Blutsverwandten als Rahmen konzentriert. In der Analyse wird die semantische Perspektive eingenommen, um Ähnlichkeiten und Unterschiede sowie tiefere soziale und kulturelle Gründe der Unterschiede in der chinesischen und der deutschen Kultur zu ermitteln. 


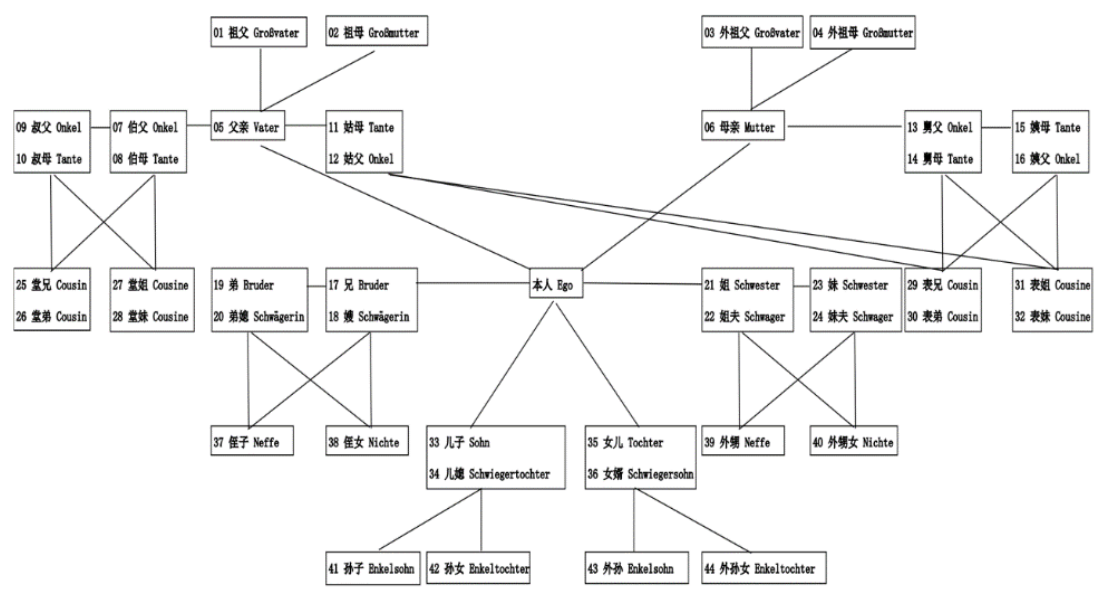

Abb. Lineare Blutsverwandtschaftsbezeichnungen (Hu 2007:92)

Die in dieser Studie verwendeten Kriterien basieren auf folgenden Verwandtschaftskriterien nach GAO (2009): Generation, Geschlecht, Alter, väterlicher/mütterlicherseits, Verwandtschaftsgrad ${ }^{10}$ und auf den Verwandtschaftskriterien nach CHEN (1990): Geschlecht, väterlicher-/mütterlicher-/ehelicherseits, Clan, Alter, Verwandtschaftsgrad, nämlich Geschlecht, Generation, väterlicher-/mütterlicherseits, Clan und Verwandtschaftsgrad.

Nach den oben genannten Klassifizierungskriterien werden die linearen Blutsverwandtschaftsbezeichnungen in der folgenden Tabelle aufgeführt:

10 Verwandtschaftsgrad bezieht sich auf die Distanz zwischen anderen Verwandten und dem Ego, zum Beispiel sind Eltern und Ego direkt miteinander verbunden, daher ist der Verwandtschaftsgrad von Eltern 1, und Geschwister sind über die Eltern mit dem Ego verbunden, sodass der Verwandtschaftsgrad 2 ist. 
Tab. 1. Verwandtschaftsbezeichnungen im Chinesischen und ihre deutschen Entsprechungen

\begin{tabular}{|c|c|c|c|c|c|c|}
\hline $\mathrm{Nr}$. & $\mathrm{VB}^{11}$ & Geschlecht & Generation & $\mathrm{v} / \mathrm{m}^{12}$ & Clan & $\mathrm{VG}^{13}$ \\
\hline \multirow[t]{2}{*}{01} & 祖父 (zǔfù) & \multirow[t]{2}{*}{$\mathrm{M}^{14}$} & aufsteigend & $\mathrm{v}$ & $\mathrm{Ja}$ & 2 \\
\hline & Großvater & & aufsteigend & $\mathrm{v} / \mathrm{m}$ & - & 2 \\
\hline \multirow[t]{2}{*}{02} & 祖母 (zǔmǔ) & \multirow[t]{2}{*}{$\mathrm{F}^{15}$} & aufsteigend & $\mathrm{v}$ & $\mathrm{Ja}$ & 2 \\
\hline & Großmutter & & aufsteigend & $\mathrm{v} / \mathrm{m}$ & - & 2 \\
\hline \multirow[t]{2}{*}{03} & $\begin{array}{l}\text { 外祖父 } \\
\text { (wàizǔfù) }\end{array}$ & \multirow[t]{2}{*}{$\mathrm{M}$} & aufsteigend & $\mathrm{m}$ & Nein & 2 \\
\hline & Großvater & & aufsteigend & $\mathrm{v} / \mathrm{m}$ & - & 2 \\
\hline \multirow[t]{2}{*}{04} & $\begin{array}{l}\text { 外祖母 } \\
\text { (wàizǔmǔ) }\end{array}$ & \multirow[t]{2}{*}{$F$} & aufsteigend & $\mathrm{m}$ & Nein & 2 \\
\hline & Großmutter & & aufsteigend & $\mathrm{v} / \mathrm{m}$ & - & 2 \\
\hline \multirow[t]{2}{*}{05} & 父亲 (füqīn) & \multirow[t]{2}{*}{$\mathrm{M}$} & aufsteigend & - & $\mathrm{Ja}$ & 1 \\
\hline & Vater & & aufsteigend & - & - & 1 \\
\hline \multirow[t]{2}{*}{06} & 母亲 (mǔqīn) & \multirow[t]{2}{*}{$\mathrm{F}$} & aufsteigend & - & $\mathrm{Ja}$ & 1 \\
\hline & Mutter & & aufsteigend & - & - & 1 \\
\hline \multirow[t]{2}{*}{07} & 伯父 (bófù) & \multirow[t]{2}{*}{ M } & $\begin{array}{l}\text { aufsteigend; } \\
\text { älter als der } \\
\text { Vater }\end{array}$ & $\mathrm{v}$ & $\mathrm{Ja}$ & 3 \\
\hline & Onkel & & aufsteigend & $\mathrm{v} / \mathrm{m}$ & - & 3 \\
\hline \multirow[t]{2}{*}{08} & 伯母 (bómǔ) & \multirow[t]{2}{*}{$\mathrm{F}$} & $\begin{array}{l}\text { aufsteigend, } \\
\text { älter als der } \\
\text { Vater }\end{array}$ & $\mathrm{v}$ & $\mathrm{Ja}$ & 3 \\
\hline & Tante & & aufsteigend & $\mathrm{v} / \mathrm{m}$ & - & 3 \\
\hline \multirow[t]{2}{*}{09} & 叔父 (shúfù) & \multirow[t]{2}{*}{ M } & $\begin{array}{l}\text { aufsteigend, } \\
\text { jünger als der } \\
\text { Vater }\end{array}$ & $\mathrm{v}$ & $\mathrm{Ja}$ & 3 \\
\hline & Onkel & & aufsteigend & $\mathrm{v} / \mathrm{m}$ & - & 3 \\
\hline \multirow[t]{2}{*}{10} & 叔母 (shúmǔ) & \multirow[t]{2}{*}{$\mathrm{F}$} & $\begin{array}{l}\text { aufsteigend, } \\
\text { jünger als der } \\
\text { Vater }\end{array}$ & $\mathrm{v}$ & $\mathrm{Ja}$ & 3 \\
\hline & Tante & & aufsteigend & $\mathrm{v} / \mathrm{m}$ & - & 3 \\
\hline \multirow[t]{2}{*}{11} & 姑母 (gūmǔ) & \multirow[t]{2}{*}{ F } & aufsteigend & $\mathrm{v}$ & Nein & 3 \\
\hline & Tante & & aufsteigend & $\mathrm{v} / \mathrm{m}$ & - & 3 \\
\hline
\end{tabular}

\footnotetext{
$11 \quad \mathrm{VB}=$ Verwandtschaftsbezeichnung

$12 \mathrm{v} / \mathrm{m}=$ väterlicherseits/mütterlicherseits

$13 \quad \mathrm{VG}=$ Verwandtschaftsgrad

$14 \quad \mathrm{M}=$ Mann

$15 \quad \mathrm{~F}=$ Frau
} 


\begin{tabular}{|c|c|c|c|c|c|c|}
\hline \multirow[t]{2}{*}{12} & 姑父 (gūfu) & \multirow[t]{2}{*}{ M } & aufsteigend & $\mathrm{v}$ & Nein & 3 \\
\hline & Onkel & & aufsteigend & $\mathrm{v} / \mathrm{m}$ & - & 3 \\
\hline \multirow[t]{2}{*}{13} & 舅父 (jiùfù) & \multirow[t]{2}{*}{$\mathrm{M}$} & aufsteigend & $\mathrm{m}$ & Nein & 3 \\
\hline & Onkel & & aufsteigend & $\mathrm{v} / \mathrm{m}$ & - & 3 \\
\hline \multirow[t]{2}{*}{14} & 舅母 (jiùmu) & \multirow[t]{2}{*}{$\mathrm{F}$} & aufsteigend & $\mathrm{m}$ & Nein & 3 \\
\hline & Tante & & aufsteigend & $\mathrm{v} / \mathrm{m}$ & - & 3 \\
\hline \multirow[t]{2}{*}{15} & 姨母 (yímǔ) & \multirow[t]{2}{*}{$\mathrm{F}$} & aufsteigend & $\mathrm{m}$ & Nein & 3 \\
\hline & Tante & & aufsteigend & $\mathrm{v} / \mathrm{m}$ & - & 3 \\
\hline \multirow[t]{2}{*}{16} & 姨夫 (yífū) & \multirow[t]{2}{*}{$\mathrm{M}$} & aufsteigend & $\mathrm{m}$ & Nein & 3 \\
\hline & Onkel & & aufsteigend & $\mathrm{v} / \mathrm{m}$ & - & 3 \\
\hline \multirow[t]{2}{*}{17} & 兄 (xiōng) & \multirow[t]{2}{*}{$\mathrm{M}$} & $\begin{array}{l}\text { dieselbe, älter } \\
\text { als das Ego }\end{array}$ & - & $\mathrm{Ja}$ & 2 \\
\hline & Bruder & & dieselbe & - & - & 2 \\
\hline \multirow[t]{2}{*}{18} & 嫂 (sǎo) & \multirow[t]{2}{*}{$\mathrm{F}$} & $\begin{array}{l}\text { dieselbe, älter } \\
\text { als das Ego }\end{array}$ & - & $\mathrm{Ja}$ & 2 \\
\hline & Schwägerin & & dieselbe & - & - & 2 \\
\hline \multirow[t]{2}{*}{19} & 弟 (di) & \multirow[t]{2}{*}{$\mathrm{M}$} & $\begin{array}{l}\text { dieselbe, jünger } \\
\text { als das Ego }\end{array}$ & - & $\mathrm{Ja}$ & 2 \\
\hline & Bruder & & dieselbe & - & - & 2 \\
\hline \multirow[t]{2}{*}{20} & 弟媳 (dìxí) & \multirow[t]{2}{*}{$\mathrm{F}$} & $\begin{array}{l}\text { dieselbe, jünger } \\
\text { als das Ego }\end{array}$ & - & $\mathrm{Ja}$ & 2 \\
\hline & Schwägerin & & dieselbe & - & - & 2 \\
\hline \multirow[t]{2}{*}{21} & 姐 (jiě) & \multirow[t]{2}{*}{$\mathrm{F}$} & $\begin{array}{l}\text { dieselbe, älter } \\
\text { als das Ego }\end{array}$ & - & Nein & 2 \\
\hline & Schwester & & dieselbe & - & - & 2 \\
\hline \multirow[t]{2}{*}{22} & 姐夫 (jiěfū) & \multirow[t]{2}{*}{$\mathrm{M}$} & $\begin{array}{l}\text { dieselbe, älter } \\
\text { als das Ego }\end{array}$ & - & Nein & 2 \\
\hline & Schwager & & dieselbe & - & - & 2 \\
\hline \multirow[t]{2}{*}{23} & 妹 (mèi) & \multirow[t]{2}{*}{ F } & $\begin{array}{l}\text { dieselbe, jünger } \\
\text { als das Ego }\end{array}$ & - & Nein & 2 \\
\hline & Schwester & & dieselbe & - & - & 2 \\
\hline \multirow[t]{2}{*}{24} & 妹夫 (mèifū) & \multirow[t]{2}{*}{ M } & $\begin{array}{l}\text { dieselbe, jünger } \\
\text { als das Ego }\end{array}$ & - & Nein & 2 \\
\hline & Schwager & & dieselbe & - & - & 2 \\
\hline \multirow[t]{2}{*}{25} & 堂兄 (tángxiōng) & \multirow[t]{2}{*}{ M } & $\begin{array}{l}\text { dieselbe, älter } \\
\text { als das Ego }\end{array}$ & $\mathrm{v}$ & $\mathrm{Ja}$ & 4 \\
\hline & Cousin & & dieselbe & $\mathrm{v} / \mathrm{m}$ & - & 4 \\
\hline \multirow[t]{2}{*}{26} & 堂弟 (tángdì) & \multirow[t]{2}{*}{$\mathrm{M}$} & $\begin{array}{l}\text { dieselbe, jünger } \\
\text { als das Ego }\end{array}$ & $\mathrm{v}$ & $\mathrm{Ja}$ & 4 \\
\hline & Cousin & & dieselbe & $\mathrm{v} / \mathrm{m}$ & - & 4 \\
\hline
\end{tabular}


Tab. 1. Verwandtschaftsbezeichnungen im Chinesischen und ihre deutschen Entsprechungen

\begin{tabular}{|c|c|c|c|c|c|c|}
\hline $\mathrm{Nr}$. & $\mathrm{VB}^{16}$ & Geschlecht & Generation & $\mathrm{v} / \mathrm{m}^{17}$ & Clan & $\mathrm{VG}^{18}$ \\
\hline \multirow[t]{2}{*}{27} & 堂姐 (tángjiě) & \multirow[t]{2}{*}{$\mathrm{F}$} & $\begin{array}{l}\text { dieselbe, älter } \\
\text { als das Ego }\end{array}$ & $\mathrm{v}$ & $\mathrm{Ja}$ & 4 \\
\hline & Cousine & & dieselbe & $\mathrm{v} / \mathrm{m}$ & - & 4 \\
\hline \multirow[t]{2}{*}{28} & 堂妹 (tángmèi) & \multirow[t]{2}{*}{$\mathrm{F}$} & $\begin{array}{l}\text { dieselbe, jünger } \\
\text { als das Ego }\end{array}$ & $\mathrm{v}$ & $\mathrm{Ja}$ & 4 \\
\hline & Cousine & & dieselbe & $\mathrm{v} / \mathrm{m}$ & - & 4 \\
\hline \multirow[t]{2}{*}{29} & 表兄 (biǎoxiōng) & \multirow[t]{2}{*}{$\mathrm{M}$} & $\begin{array}{l}\text { dieselbe, älter } \\
\text { als das Ego }\end{array}$ & $\mathrm{v} / \mathrm{m}$ & Nein & 4 \\
\hline & Cousin & & dieselbe & $\mathrm{v} / \mathrm{m}$ & - & 4 \\
\hline \multirow[t]{2}{*}{30} & 表弟 (biăodi) & \multirow[t]{2}{*}{$\mathrm{M}$} & $\begin{array}{l}\text { dieselbe, jünger } \\
\text { als das Ego }\end{array}$ & $\mathrm{v} / \mathrm{m}$ & Nein & 4 \\
\hline & Cousin & & Dieselbe & $\mathrm{v} / \mathrm{m}$ & - & 4 \\
\hline \multirow[t]{2}{*}{31} & 表姐 (biăojiě) & \multirow[t]{2}{*}{$\mathrm{F}$} & $\begin{array}{l}\text { dieselbe, älter } \\
\text { als das Ego }\end{array}$ & $\mathrm{v} / \mathrm{m}$ & Nein & 4 \\
\hline & Cousine & & dieselbe & $\mathrm{v} / \mathrm{m}$ & - & 4 \\
\hline \multirow[t]{2}{*}{32} & 表妹 (biăomèi) & \multirow[t]{2}{*}{$\mathrm{F}$} & $\begin{array}{l}\text { dieselbe, jünger } \\
\text { als das Ego }\end{array}$ & $\mathrm{v} / \mathrm{m}$ & Nein & 4 \\
\hline & Cousine & & dieselbe & $\mathrm{v} / \mathrm{m}$ & - & 4 \\
\hline \multirow[t]{2}{*}{33} & 儿子 (érzi) & \multirow[t]{2}{*}{$\mathrm{M}$} & absteigend & - & $\mathrm{Ja}$ & 1 \\
\hline & Sohn & & absteigend & - & - & 1 \\
\hline \multirow[t]{2}{*}{34} & 儿媳 (érxí) & \multirow[t]{2}{*}{$\mathrm{F}$} & absteigend & - & $\mathrm{Ja}$ & 1 \\
\hline & Schwiegertochter & & absteigend & - & - & 1 \\
\hline \multirow[t]{2}{*}{35} & 女儿 (nü'ér) & \multirow[t]{2}{*}{$\mathrm{F}$} & absteigend & - & Nein & 1 \\
\hline & Tochter & & absteigend & - & - & 1 \\
\hline \multirow[t]{2}{*}{36} & 女婿 (nǔxxù) & \multirow[t]{2}{*}{$\mathrm{M}$} & absteigend & - & Nein & 1 \\
\hline & Schwiegersohn & & absteigend & - & - & 1 \\
\hline \multirow[t]{2}{*}{37} & 侄子 (zhízi) & \multirow[t]{2}{*}{$\mathrm{M}$} & absteigend & - & $\mathrm{Ja}$ & 3 \\
\hline & Neffe & & absteigend & - & - & 3 \\
\hline \multirow[t]{2}{*}{38} & 侄女 (zhínü) & \multirow[t]{2}{*}{$\mathrm{F}$} & absteigend & - & $\mathrm{Ja}$ & 3 \\
\hline & Nichte & & absteigend & - & - & 3 \\
\hline \multirow[t]{2}{*}{39} & 外甥 (wàishēng) & \multirow[t]{2}{*}{$\mathrm{M}$} & absteigend & - & Nein & 3 \\
\hline & Neffe & & absteigend & - & - & 3 \\
\hline \multirow[t]{2}{*}{40} & $\begin{array}{l}\text { 外甥女 } \\
\text { (wàishēngnü) }\end{array}$ & \multirow[t]{2}{*}{$\mathrm{F}$} & absteigend & - & Nein & 3 \\
\hline & Nichte & & absteigend & - & - & 3 \\
\hline
\end{tabular}

$16 \quad \mathrm{VB}=$ Verwandtschaftsbezeichnung

$17 \mathrm{v} / \mathrm{m}=$ väterlicherseits/mütterlicherseits

$18 \quad \mathrm{VG}=$ Verwandtschaftsgrad 


\begin{tabular}{|l|l|l|l|l|l|l|}
\hline 41 & 孙子 (sūnzi) & \multirow{2}{*}{$\mathrm{M}$} & absteigend & - & $\mathrm{Ja}$ & 3 \\
\cline { 5 - 7 } & Enkel & & absteigend & - & - & 3 \\
\hline 42 & 孙女 (sūnnü) & \multirow{2}{*}{$\mathrm{F}$} & absteigend & - & $\mathrm{Ja}$ & 3 \\
\hline & Enkelin & & absteigend & - & - & 3 \\
\hline 43 & 外孙 (wàisūn) & \multirow{2}{*}{$\mathrm{N}$} & absteigend & - & Nein & 3 \\
\hline & Enkel & \multirow{2}{*}{$\mathrm{F}$} & absteigend & - & - & 3 \\
\hline 44 & $\begin{array}{l}\text { 外孙女 } \\
\text { (wàisūnnü) }\end{array}$ & & absteigend & - & Nein & 3 \\
\hline & Enkelin & & & & & \\
\hline & & absteigend & - & - & 3 \\
\hline
\end{tabular}

Aus der obigen Klassifizierung lassen sich einige wichtige Punkte ableiten:

1. Wie aus der obigen Tabelle hervorgeht, gibt es keinen Unterschied in Bezug auf Geschlecht und Verwandtschaftsgrad zwischen den Verwandtschaftsbezeichnungen in den beiden Sprachen.

2. Aus Sicht der Generation können die Verwandtschaftsbezeichnungen in den beiden Sprachen den Unterschied zwischen Verwandten und Ego widerspiegeln. Es ist anzumerken, dass einige chinesische Verwandtschaftsbezeichnungen den Unterschied im Alter ausdrücken können, z. B. unterscheiden sich die Geschwister durch das Alter, weil das Alter eng mit dem Status einer Person in der Familie zusammenhängt.

3. Chinesische und deutsche Verwandtschaftsbezeichnungen unterscheiden sich auch sehr in der Klassifikation nach dem Merkmal väterlicherseits/mütterlicherseits. Aus der Tabelle geht hervor, dass die deutschen Verwandtschaftsbezeichnungen nicht genau ausdrücken können, ob man es mit einem Verwandten väterlicher- oder mütterlicherseits zu tun hat. Im Gegensatz dazu können sich die chinesischen Verwandtschaftsbezeichnungen je nachdem unterscheiden, ob die Bezeichnung zur väterlichen oder mütterlichen Linie gehört.

4. Da der Begriff der Großfamilie bzw. des Clans in der deutschen Gesellschaft irrelevant ist und in der heutigen Zeit dort eine von Kernfamilien dominierte Familienstruktur überwiegt, kann sich das Kriterium Clan nicht in den Verwandtschaftsbezeichnungen widerspiegeln. Im Gegensatz dazu werden einige Verwandtschaftsbezeichnungen im Chinesischen in der Clanspalte, die als Clanverwandte hätten klassifiziert werden können, nicht als Clanverwandte gekennzeichnet, z. B. 女儿 (nü'ér, Tochter, weiblich, absteigend, Nicht-Clanverwandte, 1). Der Grund dafür ist, dass Clanverwandte Blutsverwandte unter demselben väterlichen Vorfahren einschließen, aber es muss noch eine Voraussetzung erfüllt werden, nämlich dass die weiblichen Blutsverwandten nicht verheiratet sind. Im alten China wurden weibliche Blutsverwandte nach 
ihrer Heirat in den Clan ihrer Ehemänner und nicht in die Stammbäume ihres eigenen Clans aufgenommen. Außerdem können Frauen nur als Vasallen eingetragen werden, d.h. nur deren Nachname wird aufgezeichnet. ${ }^{19}$ Aus diesem Grund sind 祖母 (zǔmǔ, Mutter des Vaters), 伯母 (bómǔ, Frau vom älteren Bruder des Vaters), 叔母 (shúmǔ, Frau vom jüngeren Bruder des Vaters) und andere Verwandte, die nicht denselben Nachnamen tragen, Clanverwandte, aber 姑母 (gūmǔ, Schwester vom Vater) und 女儿 (nü'ér, Tochter), die Verwandte mit demselben Nachnamen sind, keine Clanverwandten.

5. Im chinesischen Verwandtschaftssystem werden Cousins (Kinder von Onkeln väterlicherseits) von Cousins (Kinder von Tanten väterlicherseits und von Onkeln und Tanten mütterlicherseits) unterschieden 堂兄弟姐妹 (táng xiōngdì jiěmèi, Kinder von Onkeln väterlicherseits) und 表兄弟姐妹(biăo xiōngdì jiěmèi, Kinder von Tanten väterlicherseits und von Onkeln und Tanten mütterlicherseits). Der Unterschied zwischen den beiden Bezeichnungen beruht auch auf der Distanz nach dem patriarchalischen Konzept. Die Kinder vom väterlichen Onkel haben den gleichen Nachnamen wie das Ego und in China nehmen die Cousins vom väterlichen Onkel mit dem Ego an denselben Gedenkfeiern im Rahmen des Clan-Ahnenkults teil, deshalb ist die Verwandtschaftsbeziehung zwischen den Cousins vom väterlichen Onkel und dem Ego aus Sicht des Clans viel enger. Da 姑母 (gūmǔ, Tante von der väterlichen Seite) keine Clanverwandte ist, sind ihre Kinder auch keine Clanverwandten, daher werden sie 表兄弟姐妹 (biăo xiōngdì jiěmèi, Kinder von Tanten väterlicherseits und von Onkeln und Tanten mütterlicherseits) genannt. Weil 表兄弟姐妹 (biăo xiōngdì jiěmèi, Kinder von Tanten väterlicherseits und von Onkeln und Tanten mütterlicherseits) nicht an den Tag bringt, aus welcher Linie sie sind, werden 表兄弟姐妹 (biăo xiōngdì jiěmèi, Kinder von Tanten väterlicherseits und von Onkeln und Tanten mütterlicherseits) jedoch manchmal in drei Kategorien unterteilt: 姑表兄弟姐妹(gūbiăo xiōngdì jiěmèi, Kinder von Tanten väterlicherseits), 姨表兄弟姐妹 (yíbiăo xiōngdì jiěmèi, Kinder von Tanten mütterlicherseits) und 舅表兄弟姐妹 (jiùbiăo xiōngdì jiěmèi, Kinder von Onkeln mütterlicherseits). ${ }^{20}$ Im Vergleich zum deutschen Verwandtschaftsbezeichnungssystem ist es offensichtlich, dass die Cousins in der deutschen Bezeichnung keinen Unterschied zwischen väterlicherseits und mütterlicherseits aufweisen und es keinen Unterschied aus geschlechtlicher Perspektive gibt.

19 Vgl. https://www.sohu.com/a/147420554_648738 (12.05.2021).

20 Vgl. http://www.360doc.com/content/20/1008/07/4503649_939369510.shtml (12.05.2021). 
Aus der obigen Klassifizierung geht klar hervor, dass jede Verwandtschaftsbezeichnung im Chinesischen eine eigene Bedeutung hat, die sich eindeutig auf eine bestimmte Person oder eine kleine Gruppe beziehen kann, im deutschen Bezeichnungssystem jedoch völlig anders ist. Die Gründe sind folgende:

1. Die Verwandtschaftsbezeichnungen im Chinesischen werden vom Clan beeinflusst, während die Verwandtschaftsbezeichnungen im Deutschen überhaupt nicht vom Clan beeinflusst werden. Zum Beispiel ist es in jeder chinesischen Verwandtschaftsbezeichnung notwendig, zwischen Clanverwandtem und Nicht-Clanverwandtem zu unterscheiden, während im Falle der deutschen Verwandtschaftsbezeichnungen keine Notwendigkeit besteht, das Problem von Clanverwandten oder Nicht-Clanverwandten zu berücksichtigen. Der Grund, warum chinesische Verwandtschaftsbezeichnungen das Konzept Clan erfordern, ist, dass das alte China lange Zeit eine feudale Gesellschaft mit geringer sozialer Produktivität und unentwickelten Verkehrs- und Kommunikationsmitteln war. Menschen, die auf dem Land lebten, lebten über Generationen zusammen und entwickelten sich allmählich von losen Gruppen zu einer oder mehreren Großfamilien bzw. Clans, sodass jeder Einzelne in der Großfamilie mehr oder weniger Blutsverbindung mit anderen hatte, zum Beispiel sind die Dorfnamen auf dem Land wie 张家村 (zhāngjiācūn, Familie ZhangDorf), 王家堡 (wángjiā băo, Familie Wang-Dorf), 李家屯 (lǐjiātún, Familie Li-Dorf) usw. sehr verbreitet. Es ist sehr wichtig, die Großfamilie bzw. den Clan zu verwalten, die ein klares und genaues Verwandtschaftssystem erfordern, damit jeder seine Position in der Gruppe kennen kann (vgl. CHEN 1990:59). Lange Zeit wurde die alte chinesische Gesellschaft von 男尊女卑 (nánzūnnübēi, männliche Überlegenheit gegenüber weiblicher Unterlegenheit) beeinflusst und der Clan wurde davon auch beeinflusst, daher ist der Clan männlich zentriert und die Frauen im Clan sind die abhängige Gruppe.

2. Im Vergleich zum deutschen Verwandtschaftsbezeichnungssystem misst das chinesische der Altershierarchie große Bedeutung bei, zum Beispiel die Aufteilung von Geschwistern derselben Generation und die Aufteilung von Onkeln der väterlichen Seite in Bezug aufs Alter. Die Altershierarchie im Chinesischen hängt eng mit den Rechten, dem Status und den Pflichten innerhalb des Clans zusammen, was normalerweise als 传长不传幼 (chuán zhăng bù chuán yòu, Weitergabe an das Ältere statt Jüngere) bezeichnet wird, zum Beispiel übernimmt der älteste Sohn nach dem Tod des Vaters die Entscheidungsmacht in der Familie, aber gleichzeitig muss er sich um seine jüngeren Geschwister kümmern. Dieses von oben nach unten gerichtete, strenge und präzise Verwandtschaftssystem garantiert weitgehend den normalen Betrieb und das harmonische 
Zusammenleben der gesamten Familie. Im Vergleich dazu sind die deutschen Familien Kernfamilien, die aus Eltern und Kindern bestehen und nicht an den Clan wie chinesische Familien gebunden sind. Daher gibt es in deutschen Familien keine umständlichen Regeln wie im chinesischen Clan und es besteht keine Notwendigkeit, zwischen älteren und jüngeren Verwandten derselben Generation zu unterscheiden.

3. Im ersten Kapitel wurde ein wesentliches Merkmal der chinesischen Verwandtschaftsbezeichnungen erwähnt, nämlich der Unterschied zwischen 面称 (miàn chēng, engl. Face-to-Face addressing) und 背称 (bèi chēng, engl. backappellation). Dieser Unterschied ist auch eine Manifestation von 长幼尊卑 (zhăng yòu zūn bēi, Altershierarchie) in der chinesischen Gesellschaft. In China müssen ältere Leute mit bestimmten Verwandtschaftsbezeichnungen anstatt mit ihren Namen genannt werden. Im Gegensatz dazu hat das Familiensystem in der deutschen Gesellschaft nicht die gleichen strengen Regeln in Bezug aufs Alter wie die chinesische Familie und der Clan. Die deutsche Familienstruktur ähnelt eher einem flachen Modell, während die chinesische Familie eher einer baumartigen Struktur von oben nach unten ähnlich ist.

\section{Fazit}

Zusammenfassend lässt sich sagen, dass die Verwandtschaftsbezeichnungen im Chinesischen nicht nur eng mit dem patriarchalischen System Chinas verbunden sind, sondern auch untrennbar mit der historischen Entwicklung der chinesischen Gesellschaft. Das rigorose patriarchalische System bestimmt bis zu einem gewissen Grad, dass die chinesischen Verwandtschaftsbezeichnungen und das Verwandtschaftssystem ebenfalls rigoros und komplex sind. Zur gleichen Zeit förderte das historische Entwicklungsumfeld der geschlossenen feudalen Gesellschaft im alten China die Bildung und Stabilität dieses komplizierten Verwandtschaftsbezeichnungssystems.

Obwohl es in einigen europäischen Sprachen Unterschiede zwischen väterlichen und mütterlichen Verwandtschaftsbezeichnungen gab, wurden mit der industriellen Revolution traditionelle Familienformen in Frage gestellt und traditionelle Familienstrukturen geändert, was zu allmählichen Änderungen der Verwandtschaftsbezeichnungssysteme führte (vgl. CHEN 1990:60). Zudem begann die deutsche Gesellschaft aufgrund des ständigen Einflusses der Renaissance und der Aufklärung, Gleichheit, Freiheit und Unabhängigkeit anzustreben. Unter dem doppelten Einfluss der industriellen Revolution und der 
Renaissance ist die Familienform in Deutschland daher allmählich von der Tradition bzw. Großfamilien abgewichen und hat sich zu einem modernen bilinearen Verwandtschaftssystem entwickelt, d.h., die Bezeichnungen von Verwandten mütterlicherseits und väterlicherseits sind gleichbedeutend (vgl. JAKOBY 2008:22).

Obwohl der traditionelle chinesische Clan in der Neuzeit auch unter den Auswirkungen der industriellen Revolution und Modernisierung gelitten hat, macht die ländliche Bevölkerung immer noch einen großen Teil der Gesamtbevölkerung aus und die städtische Bevölkerung ist immer noch untrennbar mit der ländlichen Bevölkerung verbunden. Außerdem war die Dauer der Änderung noch kurz, so ist das traditionelle Verwandtschaftsbezeichnungssystem nicht vollständig zerstört, trotzdem kennen junge Leute dieses traditionelle Verwandtschaftsbezeichnungssystem immer weniger.

\section{Literatur}

Bao, Haitao / Wang, AnJie (1988): Qinshu Chengwei Cidian [Wörterbuch der Verwandtschaftsbezeichnungen]. Jilin Jiaoyu Chubanshe: Jilin.

Chen, Yueming (1990): Xiandai Hanyu Qinshu Chengwei Xitong Yiji Wenhua Yinji [Kulturelle Prägung des modernes chinesischen Verwandtschaftsbezeichnungssystems]. Hanyu Xuexi 5:57-60.

Di, Dongrui (2014): Wenhua Shijiao xia Yinghan Qinshu Chengweiyu Duibi Yanjiu [Eine vergleichende Untersuchung englischer und chinesischer Verwandtschaftsbegriffe - eine kulturorientierte Perspektive]. Wenjiao Ziliao 26:21-22.

GaO, Xiaojuan (2009): Sapir-Whorf Jiashe xia de Hanying Qinshuchengwei Yanjiu [Eine Studie über chinesische und englische Verwandtschaftsbezeichnungen im Rahmen der Sapir-Wolf-Hypothese]. Neimenggu Caijing Xueyuan Xuebao 7(2): 78-80. Hu, SHIYun (2007): Hanyu Qinshu Chengwei Yanjiu [Eine Studie über chinesische Verwandtschaftsbezeichnungen]. Shangwu Yinshuguan: Beijing.

JAKOBY, NINA (2008): (Wahl-)Verwandtschaft - Zur Erklärung verwandtschaftlichen Handelns. Wiesbaden: VS Verlag.

Murdock, GeOrge Peter (1949): Social Structure. New York: The Macmillan Company. WANG, ZHIQING (2007): Hanyu Niqinshu Chengwei Ciyu zai Richang Jiaoji zhong de Yingyong [Die Anwendung chinesischer Quasi-Verwandtschaftsbegriffe in der Alltagskommunikation]. Neimenggu Shifan Daxue Xuebao 36(6):216-217. 


\section{Internetquellen}

https://www.duden.de/rechtschreibung/Verwandtschaft abgerufen am 12.05.2021.

https://buergerliches-gesetzbuch.net/paragraph-1589 abgerufen am 12.05.2021.

https://buergerliches-gesetzbuch.net/paragraph-1590 abgerufen am 12.05.2021.

https://wenku.baidu.com/view/5b257608fl2d2af90242e6e7.html abgerufen am 12.05.2021.

http://www.360doc.com/content/20/1008/07/4503649 939369510.shtml abgerufen am 12.05.2021.

Leitfaden für die Einführungsvorlesung in Ethnosoziologie (unibe.ch) abgerufen am 23.08.2021.

Oheim - Schreibung, Definition, Bedeutung, Etymologie, Synonyme, Beispiele | DWDS abgerufen am 23.08.2021.

Muhme - Schreibung, Definition, Bedeutung, Etymologie, Synonyme, Beispiele | DWDS abgerufen am 23.08.2021.

https://www.zdic.net/hans/亲属 abgerufen am 12.05.2021.

https://cidian.18dao.net/zh-hans/zici/親屬 abgerufen am 12.05.2021.

https://www.zdic.net/hans/称谓 abgerufen am 12.05.2021.

https://www.sohu.com/a/147420554_648738 abgerufen am 12.05.2021. 